\title{
CROSSING BALANCED AND STAIR NESTED DESIGNS*
}

\author{
CÉLIA FERNANDES ${ }^{\dagger}$, PAULO RAMOS ${ }^{\dagger}$, AND JOÃO T. MEXIA ${ }^{\ddagger}$
}

\begin{abstract}
Balanced nesting is the most usual form of nesting and originates, when used singly or with crossing of such sub-models, orthogonal models. In balanced nesting we are forced to divide repeatedly the plots and we have few degrees of freedom for the first levels. If we apply stair nesting we will have plots all of the same size rendering the designs easier to apply. The stair nested designs are a valid alternative for the balanced nested designs because we can work with fewer observations, the amount of information for the different factors is more evenly distributed and we obtain good results. The inference for models with balanced nesting is already well studied. For models with stair nesting it is easy to carry out inference because it is very similar to that for balanced nesting. Furthermore stair nested designs being unbalanced have an orthogonal structure. Other alternative to the balanced nesting is the staggered nesting that is the most popular unbalanced nested design which also has the advantage of requiring fewer observations. However staggered nested designs are not orthogonal, unlike the stair nested designs. In this work we start with the algebraic structure of the balanced, the stair and the staggered nested designs and we finish with the structure of the cross between balanced and stair nested designs.
\end{abstract}

Key words. Balanced nesting, Stair nesting, Staggered nesting, Crossing, Variance components, Inference.

AMS subject classifications. $62 \mathrm{~J} 10$.

1. Introduction. Experimental designs with nesting or crossing has been discussed by several authors with different approaches, see e.g., Bailey 1, 2, Fonseca et al. 7. 8, Khuri et al. 13, Mason et al. [16, Montgomery [17] and Sahai and Ojeda 25.

The balanced nesting is the most usual form of nesting and originates, when used on itself or with crossing of such sub-models, orthogonal models. The cases when we have balanced nesting with crossing occurs surprisingly often in practice, and is frequently misunderstood. Several examples are presented for example by Bailey [1, 2], Khuri et al. 13] and Mason et al. [16].

* Received by the editors on October 10, 2011. Accepted for publication on June 6, 2012. Handling Editor: Natalia Bebiano Providencia.

${ }^{\dagger}$ ADM - Área Departamental de Matemática, ISEL - Instituto Superior de Engenharia de Lisboa, Lisboa, Portugal and CMA - Centro de Matemática e Aplicações, FCT - Faculdade de Ciências e Tecnologia, UNL - Universidade Nova de Lisboa, Caparica, Portugal

(cfernandes@adm.isel.pt,pramos@adm.isel.pt).

${ }^{\ddagger}$ CMA - Centro de Matemática e Aplicações, FCT - Faculdade de Ciências e Tecnologia, UNL Universidade Nova de Lisboa, Caparica, Portugal (jtm@fct.unl.pt) 
The balanced nested designs are orthogonal and the estimators of the variance components are independent. Although, they need a large number of observations, we are forced to divide repeatedly the plots and we have few degrees of freedom for the first levels. So the number of degrees of freedom is not evenly distributed among the factors. To overcome these disadvantages, Cox and Solomon 44 introduced a new kind of nesting, the stair nesting and its algebraic structure was proposed and studied by Fernandes et al. [6. If we apply stair nesting we will have plots all of the same size rendering the designs easier to apply. So the stair nested designs turned out to be a valid alternative for the balanced nested designs because we can work with fewer observations, the amount of information for the different factors is more evenly distributed and the number of degrees of freedom is not very different among the factors.

Another designs that appeared to overcome the disadvantages of balanced nesting are the staggered nested designs, that were introduced by Bainbridge [3]. Later, they were studied by Khattree and Naik [11, Khattree et al. [12, Naik and Khattree [18, Nelson [20, 21, 22, Ojima [23, 24] and Smith and Beverly [29]. These designs require fewer observations than the balanced nested designs and the degrees of freedom are almost the same for the different factors. However these designs are not orthogonal unlike the stair nested designs.

If we have $a(1), \ldots, a(u)$ levels for the $u$ factors that nest, in balanced nesting we have $\prod_{i=1}^{u} a(i)$ combinations of levels and in stair nesting we have $a(1)-(u-1)+$ $\sum_{i=2}^{u} a(i)$ combinations. The last factor may correspond to replicates. In the stair nested designs with $u$ factors we have $u$ steps and $a^{\bullet}(1), \ldots, a^{\bullet}(u)$ "active" levels for the $u$ factors, where $a^{\bullet}(1)=a(1)-(u-1)$ and $a^{\bullet}(j)=a(j), j=2, \ldots, u$. So the number of level combinations is $\sum_{i=1}^{u} a^{\bullet}(i)$. Each step corresponds to a one factor model. At the $h^{\text {th }}$ step we have an unique level for the first $h-1$ factors and then $a^{\bullet}(h)$ levels for the $h^{\text {th }}$ factor, which nest a single level of the following factors. The $a^{\bullet}(h)$ levels will constitute the branching associated with the $h^{\text {th }}$ factor. In staggered nesting, if we have $a^{\circ}$ (1) levels for the first factor and $u$ factors then we have $u \times a^{\circ}$ (1) combinations of levels.

In Figures 1.1, 1.2 and 1.3 we present designs with $u=3$ factors. The first one is a balanced nested design with $a(1)=4, a(2)=3$ and $a(3)=2$ levels and the number of treatments is $4 \times 3 \times 2=24$. In the second we have a stair nested design we will have $2+3+2=7$ treatments, with $a^{\bullet}(1)=2, a^{\bullet}(2)=3$ and $a^{\bullet}(3)=2$ "active" levels. The last one is a staggered nested design with $a^{\circ}(1)=4, a^{\circ}(2)=2$ and $a^{\circ}(3)=3$ levels for each factor and $3 \times 4=12$ treatments. The treatments will correspond to the factor level combinations that are considered.

For the $h^{t h}$ factor we have $c(h)=\prod_{k=1}^{h} a(k)$ levels in the balanced nested design, $c^{\bullet}(h)=(u-h)+\sum_{k=1}^{h} a^{\bullet}(k)$ levels in the stair nested design and $c^{\circ}(h)=h \times a^{\circ}(1)$ 


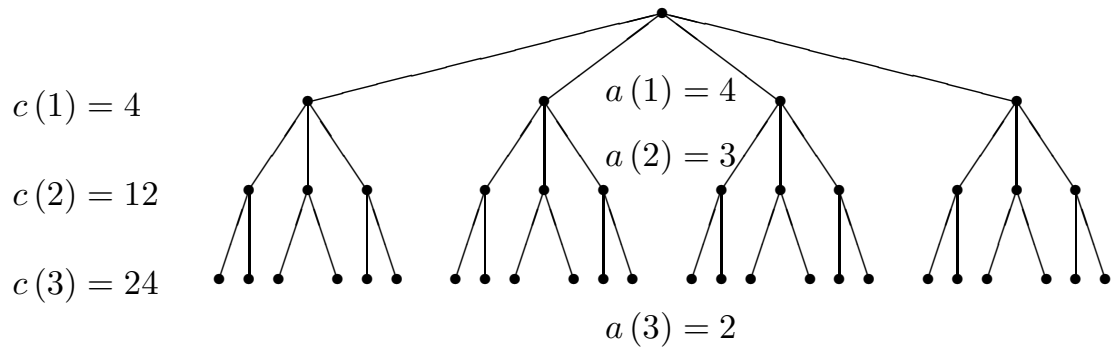

FIG. 1.1. The balanced nested design with 4, 3 and 2 levels

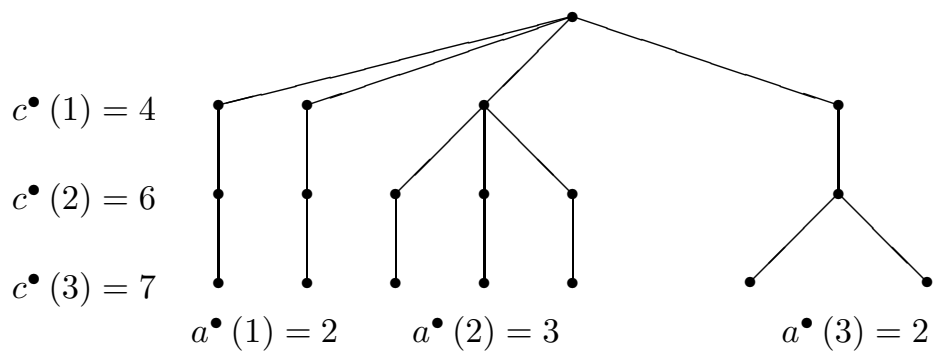

FIG. 1.2. The stair nested design with 2, 3 and 2 "active" levels

levels for the staggered nested design. So in Figure 1.1 we have $c(1)=4, c(2)=12$ and $c(3)=24$ levels; in Figure 1.2 we have $c^{\bullet}(1)=4, c^{\bullet}(2)=6$ and $c^{\bullet}(3)=7$ levels; and in Figure 1.3 we have $c^{\circ}(1)=4, c^{\circ}(2)=8$ and $c^{\circ}(3)=12$ levels.

In these three designs it is possible to obtain negative estimators for the variance components. This problem has been discussed by many authors, see e.g., Gilmour and Goos [9, Janky [10, Leone et al. [15], Nelder [19] and Searle et al. [26].

In Sections 2, 3 and 4 we present the algebraic structure of the balanced, the stair and the staggered nested designs. In Section 5 we consider the cross between balanced and stair nested designs. 


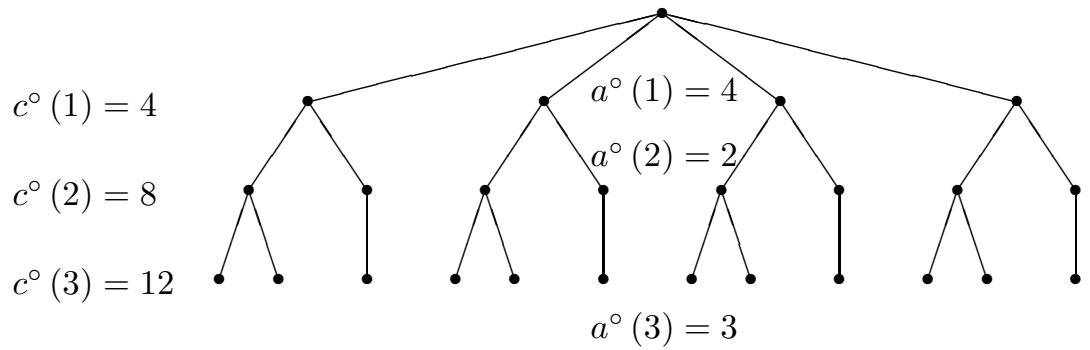

FIG. 1.3. The staggered nested design with 4, 2 and 3 levels

2. Balanced nested designs. Let there be $u$ random effect factors. The first factor will have $a(1)$ levels. If $u>1$, each level of the first factor nests $a(2)$ levels of the second factor and so on. So we will have $c(h)=\prod_{k=1}^{h} a(k)$ level combinations for the $h$ first factors, $h=1, \ldots, u$. Each of these combinations nest $b(h)=\frac{c(u)}{c(h)}$, $h=1, \ldots, u$, level combinations of the following factors. Finally we have $n=c(u)$ level combinations.

The model can be written in is canonical form as

$$
\mathbf{y}=\sum_{h=0}^{u} \mathbf{A}(h)^{\prime} \widetilde{\boldsymbol{\eta}}(h)
$$

where vectors $\widetilde{\boldsymbol{\eta}}(h)=\mathbf{A}(h) \mathbf{y}$ correspond to the effects of the factors and $\mathbf{B}^{\prime}$ is the transposed matrix of matrix B. Matrices $\mathbf{A}(h)$ are defined as

$$
\mathbf{A}(0)=\bigotimes_{k=1}^{u} \frac{1}{\sqrt{a(k)}}\left(\mathbf{1}^{a(k)}\right)^{\prime},
$$

where $\mathbf{1}^{m}$ is a column vector with $m$ components equal to 1 , and

$$
\mathbf{A}(h)=\left[\bigotimes_{k=0}^{h-1} \mathbf{I}_{a(k)}\right] \otimes \mathbf{T}_{a(h)} \otimes\left[\bigotimes_{k=h+1}^{u} \frac{1}{\sqrt{a(k)}}\left(\mathbf{1}^{a(k)}\right)^{\prime}\right],
$$

where $a(0)=1, h=1, \ldots, u, \bigotimes_{j=p+q}^{p} \mathbf{P}_{j}=\mathbf{I}_{1}, q>0, \mathbf{T}_{1}=\mathbf{I}_{1}$ and $\mathbf{I}_{m}$ the identity matrix of order $m$. Matrix $\mathbf{T}_{s}$ is obtained deleting the first row equal to $\frac{1}{\sqrt{s}}\left(\mathbf{1}^{s}\right)^{\prime}$ of a $s \times s$ orthogonal matrix, with $\mathbf{T}_{s}^{\prime} \mathbf{T}_{s}=\mathbf{I}_{s}-s^{-1} \mathbf{J}_{s}$, where $\mathbf{J}_{s}$ is the $s \times s$ matrix with all entries equal to 1 . 

matrix

The observations vector has mean vector $\boldsymbol{\mu}=\frac{1}{\sqrt{n}} 1^{n} \mu$ and variance-covariance

$$
\mathbf{V}=\sum_{h=1}^{u} \gamma(h) \mathbf{Q}(h)
$$

where $\mathbf{Q}(h)=\mathbf{A}(h)^{\prime} \mathbf{A}(h)$ and $\gamma(h)=\sum_{i=h}^{u} b(i) \sigma^{2}(i), h=1, \ldots, u$. Matrices $\mathbf{Q}(h)$ are defined as

$$
\mathbf{Q}(0)=\bigotimes_{k=1}^{u} \frac{1}{a(k)} \mathbf{J}_{a(k)}
$$

and

$$
\mathbf{Q}(h)=\left[\bigotimes_{k=0}^{h-1} \mathbf{I}_{a(k)}\right] \otimes \mathbf{K}_{a(h)} \otimes\left[\bigotimes_{k=h+1}^{u} \frac{1}{a(k)} \mathbf{J}_{a(k)}\right],
$$

where $\mathbf{K}_{a(h)}=\mathbf{T}_{a(h)}^{\prime} \mathbf{T}_{a(h)}$.

We have

$$
S(h)=\|\mathbf{A}(h) \mathbf{y}\|^{2}=\mathbf{y}^{\prime} \mathbf{A}(h)^{\prime} \mathbf{A}(h) \mathbf{y}=\mathbf{y}^{\prime} \mathbf{Q}(h) \mathbf{y}
$$

If $\mathbf{y}$ is normal then

$$
\frac{S(h)}{\gamma(h)} \sim \chi_{g(h)}^{2}
$$

with $g(h)=\operatorname{rank}[\mathbf{A}(h)]=[a(h)-1] \prod_{k=0}^{h-1} a(k), h=1, \ldots, u$ and $S(h)$ and $S(k)$ are independents for $h \neq k$. Thus we have the unbiased estimators $\widetilde{\gamma}(h)=\frac{S(h)}{g(h)}$. Then we can obtain the unbiased estimators for the variance components

$$
\tilde{\sigma}^{2}(h)=\frac{1}{b(h)}\left[\frac{S(h)}{g(h)}-\frac{S(h+1)}{g(h+1)}\right], \quad h=1, \ldots, u-1
$$

and

$$
\widetilde{\sigma}^{2}(u)=\frac{S(u)}{g(u)} .
$$

The variance of these estimators are given by

$$
\operatorname{Var}\left[\widetilde{\sigma}^{2}(h)\right]=\frac{2}{b^{2}(h)} \sum_{k=h}^{h+1} \frac{\gamma^{2}(k)}{g(k)}, \quad h=1, \ldots, u-1
$$

and

$$
\operatorname{Var}\left[\widetilde{\sigma}^{2}(u)\right]=2 \frac{\gamma^{2}(u)}{b^{2}(u)}
$$


So we have

$$
\widetilde{\operatorname{Var}}\left[\widetilde{\sigma}^{2}(h)\right]=\frac{2}{b^{2}(h)} \sum_{k=h}^{h+1} \frac{\widetilde{\gamma}^{2}(k)}{g(k)}, \quad h=1, \ldots, u-1
$$

and

$$
\widetilde{\operatorname{Var}}\left[\widetilde{\sigma}^{2}(u)\right]=2 \frac{\widetilde{\gamma}^{2}(u)}{b^{2}(u)}
$$

Furthermore we have

$$
\begin{aligned}
\mathbf{V}^{-1} & =\sum_{h=1}^{u}[\gamma(h)]^{-1} \mathbf{Q}(h), \\
\operatorname{det}(\mathbf{V}) & =\prod_{h=1}^{u}[\gamma(h)]^{g(h)}
\end{aligned}
$$

and

$$
(\mathbf{y}-\boldsymbol{\mu})^{\prime} \mathbf{V}^{-1}(\mathbf{y}-\boldsymbol{\mu})=\frac{n\left(y_{\bullet}-\mu\right)^{2}}{\gamma(1)}+\sum_{h=2}^{u} \frac{S(h)}{\gamma(h)},
$$

with $y_{\bullet}$ the general mean.

The density of $\mathbf{y}$ will be

$$
n(\mathbf{y})=\frac{\exp \left\{-\frac{1}{2}\left(\frac{n\left(y_{\bullet}-\mu\right)^{2}}{\gamma(1)}+\sum_{h=2}^{u} \frac{S(h)}{\gamma(h)}\right)\right\}}{(2 \pi)^{\frac{n}{2}} \prod_{h=1}^{u}[\gamma(h)]^{\frac{g(h)}{2}}}
$$

We may now establish

Proposition 2.1. The $y_{\bullet}$ and $S(h), h=2, \ldots, u$, are sufficient and complete statistics. The $\widetilde{\gamma}(h)$ and $\widetilde{\sigma}^{2}(h), h=1, \ldots, u$, are UMVUE.

Proof. Using the factorization theorem we see that the $y_{\bullet}$ and $S(h), h=2, \ldots, u$, are sufficient. These statistics are complete because the normal distribution belongs to the exponential family and, for these models, the parameter space contains open sets, see e.g., Lehmann 14 and Silvey [28. The last part of the thesis is now a direct consequence of the Blackwell-Lehman-Scheff theorem.

For a systematic study of this case see Khuri et al. [13]. 
TABLE 2.1

Observations.

\begin{tabular}{cccccccccccc}
\hline & 1 & & & 2 & & & 3 & & \multicolumn{3}{c}{4} \\
\hline 1 & 2 & 3 & 1 & 2 & 3 & 1 & 2 & 3 & 1 & 2 & 3 \\
\hline 3.28 & 3.52 & 2.88 & 2.46 & 1.87 & 2.19 & 2.77 & 3.74 & 2.55 & 3.78 & 4.07 & 3.31 \\
3.09 & 3.48 & 2.80 & 2.44 & 1.92 & 2.19 & 2.66 & 3.44 & 2.55 & 3.87 & 4.12 & 3.31 \\
\hline
\end{tabular}

2.1. Application. Sahai and Ojeda [25, chapter 6] report data from Snedecor and Cochran [27 on the study of the chemical content of turnip green. Four plants were sampled and from each plant, three leaves were selected. Two samples of $100 \mathrm{mg}$ were taken from each leave and, by biochemical methods, the calcium content was determined. These data are presented in Table 2.1

For this balanced nested design we have $a(1)=4, a(2)=3$ and $a(3)=2$ levels for each factor; 24 observations; $g(1)=3, g(2)=8$ and $g(3)=12$ degrees of freedom for the first, second, and third factors, respectively. Applying the theory presented in Section 2 we have

$$
\left\{\begin{array}{l}
\gamma(1)=6 \sigma^{2}(1)+2 \sigma^{2}(2)+\sigma^{2}(3) \\
\gamma(2)=2 \sigma^{2}(2)+\sigma^{2}(3) \\
\gamma(3)=\sigma^{2}(3)
\end{array}\right.
$$

and

$$
\left\{\begin{array}{l}
S(1) / g(1)=\widetilde{\gamma}(1)=2.5201 \\
S(2) / g(2)=\widetilde{\gamma}(2)=0.3288 \\
S(3) / g(3)=\widetilde{\gamma}(3)=0.0067
\end{array}\right.
$$

Hence we estimate

$$
\left\{\begin{array}{l}
\widetilde{\sigma}^{2}(1)=0.3622 \\
\widetilde{\sigma}^{2}(2)=0.1611 \\
\widetilde{\sigma}^{2}(3)=0.0067
\end{array}\right.
$$


and

$$
\left\{\begin{array}{l}
\widetilde{\operatorname{Var}}\left[\widetilde{\sigma}^{2}(1)\right]=0.1183610 \\
\widetilde{\operatorname{Var}}\left[\widetilde{\sigma}^{2}(2)\right]=0.0067577 \\
\widetilde{\operatorname{Var}}\left[\widetilde{\sigma}^{2}(3)\right]=0.0000007 .
\end{array}\right.
$$

3. Stair nested designs. The proposal in stair nested designs is to use $a^{\bullet}(1)$ levels for the first factor, combined with a single level of all other factors; then a new single level for the first factor, combined with $a^{\bullet}(2)$ new levels of the second factor, combined with a single level of all other factors; and so on. So we will have $c^{\bullet}(h)=(u-h)+\sum_{k=1}^{h} a^{\bullet}(k)$ level combinations for the $h$ first factors, $h=1, \ldots, u$.

Assuming that the observations vector $\mathbf{y}$ is normal with mean vector $\boldsymbol{\mu}$ and variance-covariance matrix $\mathbf{V}$, we put $\mathbf{y} \sim \mathcal{N}(\boldsymbol{\mu}, \mathbf{V})$. Thus the

$$
\widetilde{\boldsymbol{\eta}}_{l}(h)=\mathbf{A}_{l}(h) \mathbf{y}, \quad l=1,2 ; \quad h=1, \ldots, u
$$

will be $\mathcal{N}\left(\boldsymbol{\eta}_{l}(h), \gamma_{l}(h) \mathbf{I}_{g_{l}(h)}\right), l=1,2 ; h=1, \ldots, u$, with

$$
\boldsymbol{\eta}_{l}(h)=\mathbf{A}_{l}(h) \boldsymbol{\mu}, \quad l=1,2 ; \quad h=1, \ldots, u .
$$

Matrices $\mathbf{A}_{l}(h)$ are defined, for $h=1, \ldots, u$, as

$$
\left\{\begin{array}{l}
\mathbf{A}_{1}(h)=D\left[\mathbf{C}_{1,1}(h), \ldots, \mathbf{C}_{1, u}(h)\right] \\
\mathbf{A}_{2}(h)=D\left[\mathbf{C}_{2,1}(h), \ldots, \mathbf{C}_{2, u}(h)\right],
\end{array}\right.
$$

with

$$
\left\{\begin{array}{l}
\mathbf{C}_{1, h^{*}}(h)=\mathbf{C}_{2, h^{*}}(h)=\left(\mathbf{0}^{a^{\bullet}\left(h^{*}\right)}\right)^{\prime}, \quad h \neq h^{*} \\
\mathbf{C}_{1, h}(h)=\frac{1}{\sqrt{a^{\bullet}(h)}}\left(\mathbf{1}^{a^{\bullet}(h)}\right)^{\prime} \\
\mathbf{C}_{2, h}(h)=\mathbf{T}_{a} \cdot(h),
\end{array}\right.
$$

where $\mathbf{0}^{m}$ is a column vector with $m$ components equal to 0 . We can write $\mathbf{A}_{1}$ as

$(h)=D\left[\left(\mathbf{0}^{a^{\bullet}(1)}\right)^{\prime}, \ldots,\left(\mathbf{0}^{a^{\bullet}(h-1)}\right)^{\prime}, \frac{1}{\sqrt{a^{\bullet}(h)}}\left(\mathbf{1}^{a^{\bullet}(h)}\right)^{\prime},\left(\mathbf{0}^{a^{\bullet}(h+1)}\right)^{\prime}, \ldots,\left(\mathbf{0}^{a^{\bullet}(u)}\right)^{\prime}\right]$

and $\mathbf{A}_{2}$ as

$$
(h)=D\left[\left(\mathbf{0}^{a^{\bullet}(1)}\right)^{\prime}, \ldots,\left(\mathbf{0}^{a^{\bullet}(h-1)}\right)^{\prime}, \mathbf{T}_{a} \cdot(h),\left(\mathbf{0}^{a^{\bullet}(h+1)}\right)^{\prime}, \ldots,\left(\mathbf{0}^{a^{\bullet}(u)}\right)^{\prime}\right] .
$$


We thus have, for $h=1, \ldots, u$,

$$
\left\{\begin{array}{l}
g_{1}(h)=\operatorname{rank}\left[\mathbf{A}_{1}(h)\right]=1 \\
g_{2}(h)=\operatorname{rank}\left[\mathbf{A}_{2}(h)\right]=a^{\bullet}(h)-1
\end{array}\right.
$$

Moreover the observations vector has mean vector

$$
\boldsymbol{\mu}=\mathbf{1}^{n} \mu
$$

and variance-covariance matrix

$$
\mathbf{V}=\sum_{h=1}^{u} \sum_{l=1}^{2} \gamma_{l}(h) \mathbf{Q}_{l}(h)
$$

where

$$
\left\{\begin{array}{l}
\gamma_{1}(h)=\sum_{k=1}^{h-1} a^{\bullet}(h) \sigma^{2}(k)+\sum_{k=h}^{u} \sigma^{2}(k) \\
\gamma_{2}(h)=\sum_{k=h}^{u} \sigma^{2}(k)
\end{array}\right.
$$

and $\mathbf{Q}_{l}(h)=\mathbf{A}_{l}(h)^{\prime} \mathbf{A}_{l}(h), l=1,2$.

So, for $h=1, \ldots, u$,

$$
\left\{\begin{array}{l}
\mathbf{Q}_{1}(h)=D\left[\mathbf{B}_{1,1}(h), \ldots, \mathbf{B}_{1, u}(h)\right] \\
\mathbf{Q}_{2}(h)=D\left[\mathbf{B}_{2,1}(h), \ldots, \mathbf{B}_{2, u}(h)\right]
\end{array}\right.
$$

with

$$
\left\{\begin{array}{l}
\mathbf{B}_{1, h^{*}}(h)=\mathbf{B}_{2, h^{*}}(h)=\mathbf{0}_{a} \bullet\left(h^{*}\right), \quad h^{*} \neq h \\
\mathbf{B}_{1, h}(h)=\frac{1}{a^{\bullet}(h)} \mathbf{J}_{a} \bullet(h) \\
\mathbf{B}_{2, h}(h)=\mathbf{K}_{a} \bullet(h)
\end{array}\right.
$$

where $\mathbf{0}_{m}$ is the $m \times m$ null matrix. We can write

$$
\mathbf{Q}_{1}(h)=D\left[\mathbf{0}_{a} \bullet(1), \ldots, \mathbf{0}_{a} \bullet(h-1), \frac{1}{a \bullet(h)} \mathbf{J}_{a} \bullet(h), \mathbf{0}_{a} \bullet(h+1), \ldots, \mathbf{0}_{a} \bullet(u)\right]
$$

and

$$
\mathbf{Q}_{2}(h)=D\left[\mathbf{0}_{a} \bullet(1), \ldots, \mathbf{0}_{a} \bullet(h-1), \mathbf{K}_{a} \bullet(h), \mathbf{0}_{a} \bullet(h+1), \ldots, \mathbf{0}_{a} \bullet(u)\right]
$$


We have that $\boldsymbol{\eta}_{2}(h)=\mathbf{0}^{g_{2}(h)}, h=1, \ldots, u$, and that the cross of covariance matrices of the $\widetilde{\boldsymbol{\eta}}_{l}(h), l=1,2 ; h=1, \ldots, u$, are null so these vectors will be independent.

We will center inference on the variance components using the fact that the

$$
S(h)=\left\|\widetilde{\boldsymbol{\eta}}_{2}(h)\right\|^{2}=\left\|\mathbf{A}_{2}(h) \mathbf{y}\right\|^{2}=\mathbf{y}^{\prime} \mathbf{A}_{2}(h)^{\prime} \mathbf{A}_{2}(h) \mathbf{y}=\mathbf{y}^{\prime} \mathbf{Q}_{2}(h) \mathbf{y},
$$

with $h=1, \ldots, u$. Since $\mathbf{y}$ is normal then

$$
\frac{S(h)}{\gamma_{2}(h)} \sim \chi_{g_{2}(h)}^{2},
$$

with $h=1, \ldots, u$, and $S(h)$ and $S(k)$ are independent for $h \neq k$. Thus we have the unbiased estimators

$$
\widetilde{\gamma_{2}}(h)=\frac{S(h)}{g_{2}(h)}, \quad h=1, \ldots, u
$$

from which we get

$$
\left\{\begin{array}{l}
\widetilde{\sigma}^{2}(u)=\widetilde{\gamma_{2}}(u) \\
\widetilde{\sigma}^{2}(h)=\widetilde{\gamma_{2}}(h)-\widetilde{\gamma_{2}}(h+1), \quad h=1, \ldots, u-1 .
\end{array}\right.
$$

The variance of these estimators are given by

$$
\operatorname{Var}\left[\widetilde{\sigma}^{2}(h)\right]=2 \sum_{k=h}^{h+1} \frac{\gamma_{2}^{2}(k)}{a(k)-1}, \quad h=1, \ldots, u-1
$$

and

$$
\operatorname{Var}\left[\widetilde{\sigma}^{2}(u)\right]=2 \frac{\gamma_{2}^{2}(u)}{a(u)-1} .
$$

So we have

$$
\widetilde{\operatorname{Var}}\left[\widetilde{\sigma}^{2}(h)\right]=2 \sum_{k=h}^{h+1} \frac{\widetilde{\gamma}_{2}^{2}(k)}{a(k)-1}, \quad h=1, \ldots, u-1
$$

and

$$
\widetilde{\operatorname{Var}}\left[\widetilde{\sigma}^{2}(u)\right]=2 \frac{\widetilde{\gamma}_{2}^{2}(u)}{a(u)-1}
$$

Furthermore

$$
\left\{\begin{array}{l}
\mathbf{V}^{-1}=\sum_{h=1}^{u} \sum_{l=1}^{2}\left[\gamma_{l}(h)\right]^{-1} \mathbf{Q}_{l}(h) \\
\operatorname{det}(\mathbf{V})=\prod_{h=1}^{u} \prod_{l=1}^{2}\left[\gamma_{l}(h)\right]^{g_{l}(h)}
\end{array}\right.
$$


TABLE 3.1

Selected observations.

\begin{tabular}{|c|c|c|c|c|c|c|c|c|c|c|c|}
\hline & 1 & & & 2 & & & 3 & & & 4 & \\
\hline 1 & 2 & 3 & 1 & 2 & 3 & 1 & 2 & 3 & 1 & 2 & 3 \\
\hline & & & & & & 2.77 & & 2.55 & 3.78 & & \\
\hline 3.09 & & & & 1.92 & & & 3.44 & & 3.87 & & \\
\hline
\end{tabular}

and since that

$$
\begin{aligned}
(\mathbf{y}-\boldsymbol{\mu})^{\prime} \mathbf{V}^{-1}(\mathbf{y}-\boldsymbol{\mu}) & =\sum_{h=1}^{u} \sum_{l=1}^{2} \frac{(\mathbf{y}-\boldsymbol{\mu})^{\prime}\left[\mathbf{A}_{l}(h)\right]^{\prime} \mathbf{A}_{l}(h)(\mathbf{y}-\boldsymbol{\mu})}{\gamma_{l}(h)}= \\
& =\sum_{h=1}^{u} \frac{\left\|\widetilde{\boldsymbol{\eta}}_{1}(h)-\boldsymbol{\eta}_{1}(h)\right\|^{2}}{\gamma_{1}(h)}+\sum_{h=1}^{u} \frac{S(h)}{\gamma_{2}(h)}
\end{aligned}
$$

the density of $\mathbf{y}$ will be

$$
n(\mathbf{y})=\frac{\exp \left\{-\frac{1}{2}\left[\sum_{h=1}^{u} \frac{\left\|\tilde{\boldsymbol{n}}_{1}(h)-\boldsymbol{\eta}_{1}(h)\right\|^{2}}{\gamma_{1}(h)}+\sum_{h=1}^{u} \frac{S(h)}{\gamma_{2}(h)}\right]\right\}}{(2 \pi)^{\frac{n}{2}} \prod_{h=1}^{u} \prod_{l=1}^{2}\left[\gamma_{l}(h)\right]^{\frac{g_{l}(h)}{2}}} .
$$

Using the factorization theorem we see that $\widetilde{\boldsymbol{\eta}}_{1}(h)$ and $S(h), h=1, \ldots, u$, are sufficient. According to the Rao-Blackwell theorem the estimators should, as we previously show, be function of the sufficient statistics.

3.1. Application. In this application we will use the data presented in Subsection 2.1. For the stair nested design we have $a^{\bullet}(1)=2$, $a^{\bullet}(2)=3$ and $a^{\bullet}(3)=2$ "active" levels for the first, second, and third factors; 7 observations; $g(1)=1$, $g(2)=2$ and $g(3)=1$ degrees of freedom for each factor. Applying the theory presented in Section 3 we have

$$
\left\{\begin{array}{l}
\gamma(1)=\sigma^{2}(1)+\sigma^{2}(2)+\sigma^{2}(3) \\
\gamma(2)=\sigma^{2}(2)+\sigma^{2}(3) \\
\gamma(3)=\sigma^{2}(3)
\end{array}\right.
$$

In Table 3.1 we show 7 random observations selected from the original 24 to give a stair nested design. 
These data give

$$
\left\{\begin{array}{l}
S(1) / g(1)=\widetilde{\gamma}(1)=0.9248 \\
S(2) / g(2)=\widetilde{\gamma}(2)=0.2149 \\
S(3) / g(3)=\widetilde{\gamma}(3)=0.0041
\end{array}\right.
$$

and hence we estimate

$$
\left\{\begin{array}{l}
\tilde{\sigma}^{2}(1)=0.7099 \\
\widetilde{\sigma}^{2}(2)=0.2109 \\
\widetilde{\sigma}^{2}(3)=0.0041
\end{array}\right.
$$

and

$$
\left\{\begin{array}{l}
\widetilde{\operatorname{Var}}\left[\widetilde{\sigma}^{2}(1)\right]=1.7566900 \\
\widetilde{\operatorname{Var}}\left[\widetilde{\sigma}^{2}(2)\right]=0.0462148 \\
\widetilde{\operatorname{Var}}\left[\widetilde{\sigma}^{2}(3)\right]=0.0000328
\end{array}\right.
$$

4. Staggered nested designs. The staggered nested designs belong to the class of unbalanced nested designs and were proposed and named by Bainbridge 3 . It is the most popular unbalanced nested design in practical fields, because it has a simple structure and each sum of squares in the analysis of variance has almost the same number of degrees of freedom. For the first factor we have $a^{\circ}(1)-1$ degrees of freedom and $a^{\circ}(1)$ degrees of freedom for the remaining factors, where $a^{\circ}(1)$ is the number of levels for the first factor. However this design is not orthogonal and the sums of squares are not independents. In a staggered nested design we have $a^{\circ}(1)$ experimental units. In Figure 4.1 we present an experiment unit for a staggered nested design with three factors. For $i=1, \ldots, a^{\circ}(1)$, the $i^{t h}$ experimental unit of a threefactor staggered nested design has three observations: $y_{i 1}, y_{i 2}$ and $y_{i 3}$. If we have $u$ factors then we have $u$ observations, $y_{i j}$, with $i=1, \ldots, a^{\circ}(1)$ and $j=1, \ldots, u$, for the $i^{t h}$ experimental unit.

In this work we consider a three-factor staggered nested design. We have the 


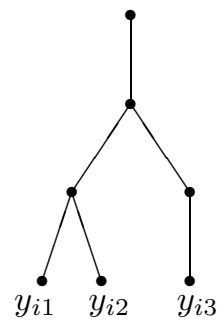

FIG. 4.1. The $i^{\text {th }}$ experimental unit of the three-factor staggered nested design

sums of squares:

$$
\left\{\begin{array}{l}
S(1)=\frac{1}{3} \sum_{i=1}^{a^{\circ}(1)}\left(\sum_{j=1}^{3} y_{i j}\right)^{2}-\frac{1}{3 a^{\circ}(1)}\left(\sum_{i=1}^{a^{\circ}(1)} \sum_{j=1}^{3} y_{i j}\right)^{2} \\
S(2)=\frac{1}{6} \sum_{i=1}^{a^{\circ}(1)}\left(y_{i 1}+y_{i 2}-2 y_{i 3}\right)^{2} \\
S(3)=\frac{1}{2} \sum_{i=1}^{a^{\circ}(1)}\left(y_{i 1}-y_{i 2}\right)^{2}
\end{array}\right.
$$

with the degrees of freedom

$$
\left\{\begin{array}{l}
g(1)=a^{\circ}(1)-1 \\
g(2)=g(3)=a^{\circ}(1) .
\end{array}\right.
$$

The canonical variance components are given by

$$
\left\{\begin{array}{l}
\gamma(1)=3 \sigma^{2}(1)+\frac{5}{3} \sigma^{2}(2)+\sigma^{2}(3) \\
\gamma(2)=\frac{4}{3} \sigma^{2}(2)+\sigma^{2}(3) \\
\gamma(3)=\sigma^{2}(3)
\end{array}\right.
$$

and we have

$$
\left\{\begin{array}{l}
\widetilde{\gamma}(1)=S(1) / g(1) \\
\widetilde{\gamma}(2)=S(2) / g(2) \\
\widetilde{\gamma}(3)=S(3) / g(3)
\end{array}\right.
$$


TABLE 4.1

Selected observations.

\begin{tabular}{|c|c|c|c|c|c|c|c|c|c|c|c|}
\hline & 1 & & & 2 & & & 3 & & & 4 & \\
\hline 1 & 2 & 3 & 1 & 2 & 3 & 1 & 2 & 3 & 1 & 2 & 3 \\
\hline 3.28 & 3.52 & & & 1.87 & & & & 2.55 & 3.78 & & 3.31 \\
\hline 3.09 & & & & 1.92 & 2.19 & 2.66 & & 2.55 & 3.87 & & \\
\hline
\end{tabular}

Then we can obtain the estimators for the usual variance components

$$
\left\{\begin{array}{l}
\widetilde{\sigma}^{2}(1)=\frac{1}{12}[4 \widetilde{\gamma}(1)-5 \widetilde{\gamma}(2)+\widetilde{\gamma}(3)] \\
\widetilde{\sigma}^{2}(2)=\frac{3}{4}[\widetilde{\gamma}(2)-\widetilde{\gamma}(3)] \\
\widetilde{\sigma}^{2}(3)=\widetilde{\gamma}(3)
\end{array}\right.
$$

and the variance of these estimators are given by

$$
\left\{\begin{aligned}
\operatorname{Var}\left[\widetilde{\sigma}^{2}(1)\right]= & \frac{2}{a^{\circ}(1)}\left[\frac{13}{72} \sigma^{4}(3)+\frac{25}{54} \sigma^{2}(3) \sigma^{2}(2)+\frac{5}{27} \sigma^{4}(2)\right]+ \\
& +\frac{2}{a^{\circ}(1)-1}\left[\frac{1}{3} \sigma^{2}(3)+\frac{5}{9} \sigma^{2}(2)+\sigma^{2}(1)\right]^{2} \\
\operatorname{Var}\left[\widetilde{\sigma}^{2}(2)\right]= & \frac{2}{a^{\circ}(1)}\left[\frac{9}{8} \sigma^{4}(3)+\frac{3}{2} \sigma^{2}(3) \sigma^{2}(2)+\sigma^{4}(2)\right] \\
\operatorname{Var}\left[\widetilde{\sigma}^{2}(3)\right]= & \frac{2}{a^{\circ}(1)} \sigma^{4}(3) .
\end{aligned}\right.
$$

For a systematic study of this case see Ojima [23].

4.1. Application. In this application we will use the data presented in Subsection [2.1. For the staggered nested design we have $a^{\circ}(1)=4, a^{\circ}(2)=2$ and $a^{\circ}(3)=3$ levels for the first, second, and third factors; 12 observations; $g(1)=3$, $g(2)=g(3)=4$ degrees of freedom for each factor. We have

$$
\left\{\begin{array}{l}
\gamma(1)=3 \sigma^{2}(1)+\frac{5}{3} \sigma^{2}(2)+\sigma^{2}(3) \\
\gamma(2)=\frac{4}{3} \sigma^{2}(2)+\sigma^{2}(3) \\
\gamma(3)=\sigma^{2}(3)
\end{array}\right.
$$

In Table 4.1 we show 12 random observations selected from the original 24 to give a staggered nested design. 
These data give

$$
\left\{\begin{array}{l}
S(1) / g(1)=\widetilde{\gamma}(1)=1.6439 \\
S(2) / g(2)=\widetilde{\gamma}(2)=0.0794 \\
S(3) / g(3)=\widetilde{\gamma}(3)=0.0058
\end{array}\right.
$$

and hence we estimate

$$
\left\{\begin{array}{l}
\tilde{\sigma}^{2}(1)=0.5153 \\
\tilde{\sigma}^{2}(2)=0.0552 \\
\tilde{\sigma}^{2}(3)=0.0058
\end{array}\right.
$$

and

$$
\left\{\begin{array}{l}
\widetilde{\operatorname{Var}}\left[\widetilde{\sigma}^{2}(1)\right]=0.2005270 \\
\widetilde{\operatorname{Var}}\left[\widetilde{\sigma}^{2}(2)\right]=0.0017840 \\
\widetilde{\operatorname{Var}}\left[\widetilde{\sigma}^{2}(3)\right]=0.0000170
\end{array}\right.
$$

5. Crossing balanced and stair nested designs. In this section we intend to study the cross between balanced and stair nested designs. In this designs we consider $L=2$ groups with $u_{1}=2$ and $u_{2}=2$ factors in each group, respectively, and $r$ replicates. In the first group we have a balanced nested design and in the second group we have a stair nested design. In the balanced nested design we have two factors with $a_{1}(1)$ and $a_{1}$ (2) levels and in the stair nested design we have two factors with $a_{2}(1)$ and $a_{2}(2)$ "active" levels. So we have $n_{1}=\prod_{i=1}^{2} a_{1}(i)$ treatments in the first group and $n_{2}=\sum_{i=1}^{2} a_{2}(i)$ treatments in the second group. Finally, we have $n=n_{1} \times n_{2} \times r$ observations.

If we consider the intervenient factors, the parameters will be:

- the general mean value;

- the effects of the different factors;

- the interactions between levels of factors in distinct groups.

These parameters will be associated to vectors $\mathbf{h}=\left(h_{1}, h_{2}\right)$ of

$$
\Gamma=\left\{\mathbf{h}: 0 \leq h_{1} \leq 2 ; \quad 0 \leq h_{2} \leq 2\right\}
$$


For the first group we can write the model in is canonical form as

$$
\mathbf{y}_{1}=\sum_{k_{1}=0}^{2} \mathbf{A}_{1}\left(k_{1}\right)^{\prime} \widetilde{\boldsymbol{\eta}}\left(k_{1}\right)
$$

with $\widetilde{\boldsymbol{\eta}}\left(k_{1}\right)=\mathbf{A}_{1}\left(k_{1}\right) \mathbf{y}_{1}$.

Matrices $\mathbf{A}_{1}\left(k_{1}\right)$ are defined as

$$
\left\{\begin{array}{l}
\mathbf{A}_{1}(0)=\frac{1}{\sqrt{a_{1}(1)}}\left(\mathbf{1}^{a_{1}(1)}\right)^{\prime} \otimes \frac{1}{\sqrt{a_{1}(2)}}\left(\mathbf{1}^{a_{1}(2)}\right)^{\prime} \\
\mathbf{A}_{1}(1)=\mathbf{T}_{a_{1}(1)} \otimes \frac{1}{\sqrt{a_{1}(2)}}\left(\mathbf{1}^{a_{1}(2)}\right)^{\prime} \\
\mathbf{A}_{1}(2)=\mathbf{I}_{a_{1}(1)} \otimes \mathbf{T}_{a_{1}(2)} .
\end{array}\right.
$$

For the second group we have

$$
\widetilde{\boldsymbol{\eta}}_{j}\left(k_{2}\right)=\mathbf{A}_{2, j}\left(k_{2}\right) \mathbf{y}_{2},
$$

for $j=1,2$ and $k_{2}=1,2$.

Matrices $\mathbf{A}_{2, j}\left(k_{2}\right)$ are defined as

$$
\left\{\begin{array}{l}
\mathbf{A}_{2,1}(1)=D\left[\frac{1}{\sqrt{a_{2}(1)}}\left(\mathbf{1}^{a_{2}(1)}\right)^{\prime},\left(\mathbf{0}^{a_{2}(2)}\right)^{\prime}\right] \\
\mathbf{A}_{2,2}(1)=D\left[\mathbf{T}_{a_{2}(1)},\left(\mathbf{0}^{a_{2}(2)}\right)^{\prime}\right] \\
\mathbf{A}_{2,1}(2)=D\left[\left(\mathbf{0}^{a_{2}(1)}\right)^{\prime}, \frac{1}{\sqrt{a_{2}(2)}}\left(\mathbf{1}^{a_{2}(2)}\right)^{\prime}\right] \\
\mathbf{A}_{2,2}(2)=D\left[\left(\mathbf{0}^{a_{2}(1)}\right)^{\prime}, \mathbf{T}_{a_{2}(2)}\right] .
\end{array}\right.
$$

When we cross both groups we obtain the model in is canonical form as

$$
\mathbf{y}=\sum_{\mathbf{h} \in \Gamma} \mathbf{A}(\mathbf{h})^{\prime} \boldsymbol{\eta}(\mathbf{h})+\mathbf{A}^{\perp} \mathbf{e} .
$$

Matrices $\mathbf{A}(\mathbf{h})$ are defined as:

- matrix $\mathbf{A}(0,0)=\mathbf{A}_{1}(0,1)+\mathbf{A}_{1}(0,2)$ correspond to the general mean value;

- matrix $\mathbf{A}(i, 0)=\mathbf{A}_{1}(i, 1)+\mathbf{A}_{1}(i, 2), i=1,2$, correspond to the effect of the $i^{\text {th }}$ factor of the $1^{\text {st }}$ group; 
- matrix $\mathbf{A}(0, l)=\mathbf{A}_{2}(0, l), l=1,2$, correspond to the effect of the $l^{\text {th }}$ factor of the $2^{\text {nd }}$ group;

- matrix $\mathbf{A}(i, 1)=\mathbf{A}_{2}(i, 1), i=1,2$, correspond to the interaction between the $i^{\text {th }}$ factor of the $1^{\text {st }}$ group and the $1^{\text {st }}$ factor of the $2^{\text {nd }}$ group;

- matrix $\mathbf{A}(i, 2)=\mathbf{A}_{2}(i, 2), i=1,2$, correspond to the interaction between the $i^{\text {th }}$ factor of the $1^{\text {st }}$ group and the $2^{\text {nd }}$ factor of the $2^{\text {nd }}$ group,

with matrices

$$
\mathbf{A}_{j}\left(k_{1}, k_{2}\right)=\mathbf{A}_{1}\left(k_{1}\right) \otimes \mathbf{A}_{2, j}\left(k_{2}\right) \otimes \frac{1}{\sqrt{r}}\left(\mathbf{1}^{r}\right)^{\prime},
$$

with $j=1,2 ; k_{1}=0,1,2$ and $k_{2}=1,2$. Matrix $\mathbf{A}^{\perp}$ is defined as

$$
\mathbf{A}^{\perp}=\mathbf{I}_{n_{1} \times n_{2}} \otimes \mathbf{T}_{r} .
$$

We have a random effects model and we assume that $\mathbf{y}$ is normal with mean vector $\boldsymbol{\mu}$ and variance-covariance matrix

$$
\mathbf{V}=\sum_{\mathbf{h} \in \Gamma} \gamma(\mathbf{h}) \mathbf{Q}(\mathbf{h})+\sigma^{2} \mathbf{Q}^{\perp}
$$

where $\mathbf{Q}(\mathbf{h})=\mathbf{A}(\mathbf{h})^{\prime} \mathbf{A}(\mathbf{h})$ and $\mathbf{Q}^{\perp}=\left(\mathbf{A}^{\perp}\right)^{\prime} \mathbf{A}^{\perp}$. We put $\mathbf{y} \sim \mathcal{N}(\boldsymbol{\mu}, \mathbf{V})$. So we have

$$
\widetilde{\boldsymbol{\eta}}(\mathbf{h})=\mathbf{A}(\mathbf{h}) \mathbf{y}
$$

and

$$
\widetilde{\boldsymbol{\eta}}^{\perp}=\mathbf{A}^{\perp} \mathbf{y}
$$

where $\widetilde{\boldsymbol{\eta}}(\mathbf{h}) \sim \mathcal{N}\left[\mathbf{0}, \gamma(\mathbf{h}) \mathbf{I}_{g(\mathbf{h})}\right]$ with $g(\mathbf{h})=\operatorname{rank}[\mathbf{A}(\mathbf{h})], \widetilde{\boldsymbol{\eta}}^{\perp} \sim \mathcal{N}\left[\mathbf{0}, \sigma^{2} \mathbf{I}_{g}\right]$ with $g=\operatorname{rank}\left[\mathbf{A}^{\perp}\right]$ and

$$
\gamma(\mathbf{h})=\sigma^{2}+\sum_{\mathbf{k}: \mathbf{h} \leq \mathbf{k}} \sigma^{2}(\mathbf{k}), \quad \mathbf{k} \in \Gamma .
$$

Taking $\Theta(\mathbf{k})=\left\{\mathbf{h}: k_{j} \leq h_{j} \leq \min \left\{u_{j} ; k_{j}+1\right\}, \quad j=1,2\right\}$ we have the

Proposition 5.1. We have

$$
\sigma^{2}(\mathbf{k})=\sum_{\mathbf{h} \in \Theta(\mathbf{k})}(-1)^{n(\mathbf{k}, \mathbf{h})} \gamma(\mathbf{h}),
$$

with $n(\mathbf{k}, \mathbf{h})$ the number of components of $\mathbf{k}$ lesser than homologous components of h. 
Proof. From the second member of the equality we get

$$
\sum_{\mathbf{h} \in \Theta(\mathbf{k})}(-1)^{n(\mathbf{k}, \mathbf{h})} \gamma(\mathbf{h})=\sum_{\mathbf{h} \in \Theta(\mathbf{k})}(-1)^{n(\mathbf{k}, \mathbf{h})}\left[\sigma^{2}+\sum_{\mathbf{h}^{\prime}: \mathbf{h} \leq \mathbf{h}^{\prime}} \sigma^{2}\left(\mathbf{h}^{\prime}\right)\right] .
$$

So, $\sigma^{2}\left(\mathbf{h}^{\prime}\right)$ has coefficients $(-1)^{n(\mathbf{k}, \mathbf{h})}$ in corresponding terms of $\mathbf{h}$ such that $\mathbf{h} \in$ $\Theta(\mathbf{k})$ and $\mathbf{h} \leq \mathbf{h}^{\prime}$. As $\mathbf{k} \leq \mathbf{h} \leq \mathbf{h}^{\prime}$, if $\mathbf{h}^{\prime}=\mathbf{k}$ there will be a single vector $\mathbf{h}$ that will be $\mathbf{h}=\mathbf{k}$ for which $n(\mathbf{k}, \mathbf{h})=0$ and, consequently $(-1)^{n(\mathbf{k}, \mathbf{k})}=1$, so $\sigma^{2}(\mathbf{k})$ will have coefficient 1 . If $\mathbf{h}^{\prime} \neq \mathbf{k}$ we will have $\mathbf{h}^{\prime}>\mathbf{k}$. Let $\mathcal{C}\left(\mathbf{h}^{\prime}\right)$ be the set of indexes of the components of $\mathbf{h}^{\prime}$ that exceed the homologous components of $\mathbf{k}$.

For $\mathcal{C} \subseteq \mathcal{C}\left(\mathbf{h}^{\prime}\right)$ there will be one and only one $\mathbf{h} \in \Theta(\mathbf{k})$ such that $\mathcal{C}(\mathbf{h})=\mathcal{C}$. The coefficient of $\sigma^{2}\left(\mathbf{h}^{\prime}\right)$ in the term of $\mathbf{h}$ will be $(-1)^{n(\mathbf{k}, \mathbf{h})}$. Now there are $\left(\begin{array}{c}n\left(\mathbf{k}, \mathbf{h}^{\prime}\right) \\ t\end{array}\right)$ sets $\mathcal{C} \subseteq \mathcal{C}\left(\mathbf{h}^{\prime}\right)$ with $\sharp(\mathcal{C})=t$, so the coefficient of $\sigma^{2}\left(\mathbf{h}^{\prime}\right)$ will be

$$
\sum_{t=0}^{n\left(\mathbf{k}, \mathbf{h}^{\prime}\right)}\left(\begin{array}{c}
n\left(\mathbf{k}, \mathbf{h}^{\prime}\right) \\
t
\end{array}\right)(-1)^{t}=0 .
$$

Reasoning as above we see that the coefficient of $\sigma^{2}$ is

$$
\sum_{t=0}^{2}\left(\begin{array}{l}
2 \\
t
\end{array}\right)(-1)^{t}=0
$$

which establishes the thesis.

We have the sums of squares

$$
\mathbf{S}(\mathbf{h})=\|\mathbf{A}(\mathbf{h}) \mathbf{y}\|^{2} \sim \gamma(\mathbf{h}) \chi_{g(\mathbf{h})}^{2}, \quad \mathbf{h} \in \Gamma
$$

and

$$
\mathrm{S}=\left\|\mathbf{A}^{\perp} \mathbf{y}\right\|^{2} \sim \sigma^{2} \chi_{g}^{2} .
$$

Thus we have the unbiased estimators

$$
\widetilde{\gamma}(\mathbf{h})=\frac{S(\mathbf{h})}{g(\mathbf{h})}
$$

and

$$
\tilde{\sigma}^{2}=\frac{S}{g} .
$$

Then we can obtain the unbiased estimators for the variance components

$$
\widetilde{\sigma}^{2}(\mathbf{k})=\sum_{\mathbf{h} \in \Theta(\mathbf{k})}(-1)^{n(\mathbf{k}, \mathbf{h})} \widetilde{\gamma}(\mathbf{h}) .
$$


Furthermore we have

$$
\begin{aligned}
\operatorname{det}[\mathbf{V}] & =\prod_{\mathbf{h} \in \Gamma}[\gamma(\mathbf{h})]^{g(\mathbf{h})}\left(\sigma^{2}\right)^{g^{\perp}} \\
\mathbf{V}^{-1} & =\sum_{\mathbf{h} \in \Gamma} \gamma^{-1}(\mathbf{h}) \mathbf{Q}(\mathbf{h})+\frac{1}{\sigma^{2}} \mathbf{Q}^{\perp}
\end{aligned}
$$

The normal density of $\mathbf{y}$ will be

$$
n(\mathbf{y})=\frac{\exp \left\{-\frac{1}{2}\left[\frac{\|\mathbf{A}(0,0) \mathbf{y}-\mathbf{A}(0,0) \boldsymbol{\mu}\|^{2}}{\gamma(0,0)}+\sum_{\mathbf{h} \in \Gamma \backslash\{\mathbf{0}\}} \frac{\mathbf{S}(\mathbf{h})}{\gamma(\mathbf{h})}+\frac{S}{g}\right]\right\}}{(2 \pi)^{\frac{n}{2}} \prod_{\mathbf{h} \in \Gamma}[\gamma(\mathbf{h})]^{\frac{g(\mathbf{h})}{2}}\left(\sigma^{2}\right)^{\frac{g \perp}{2}}} .
$$

Using the factorization theorem we see that $\widetilde{\eta}(0,0)=\mathbf{A}(0,0) \mathbf{y}, \mathbf{S}(\mathbf{h})$, for $\mathbf{h} \in$ $\Gamma \backslash\{\mathbf{0}\}$, and $\mathrm{S}$ are a set of sufficient statistics. According to the Rao-Blackwell theorem, estimators should, as we previously show, be function of the sufficient statistics.

5.1. Application. Fernandes [5] give an example of a study about the problem of genetic homogeneity of the grapevine castes. The grapevines are produced through cloning. Clones with a possible common ancestor constitute a caste. Although the castes should be genetically homogeneous, some farmers consider that this is not always true. Two groups of three clones, obtained in different regions for "Touriga Nacional" were cultivated jointly. In this experiment we have a model with four random factors, the location inside the field, the degree of humidity on the ground, the origin of the plants and the clone and three replicates were considered. The grapevines were planted in a rectangular grid and in each row of the grid a clone was planted. The production in kilos per grapevine are presented in Tables 5.1 and 5.2 .

We have a cross of balanced nesting with $3 \times 5 \times 2 \times 3=90$ treatments. On one side we have the origin, $\mathrm{O}$, with two levels, that nest the clone, $\mathrm{C}$, with three levels. On the other side we have the localization on the field, L, with three levels, that nest the degree of humidity, H, with five levels. Finally we have three replicates for each combination of factors. So we have $n=n_{1} \times n_{2} \times r=6 \times 15 \times 3=270$ observations.

In this work we intend to study the cross between two groups in which, in the first group we have a balanced nested design, and in the second group we have a stair nested design. So we have $L=2$ groups with $u_{1}=2$ and $u_{2}=2$ factors in each group and $r=3$ replicates. In the first group we have balanced nesting with two factors, the origin, $\mathrm{O}$, and the clone, $\mathrm{C}$, and in the second group we have stair nesting with two factors, the localization in the field, L, and the degree of humidity, H. The number of levels for the different factors are $a_{1}(1)=2$ and $a_{1}(2)=3$ levels for the 
TABLE 5.1

Production in $\mathrm{kg}$ per plant - observations.

\begin{tabular}{|c|c|c|c|c|c|c|c|c|c|c|c|}
\hline & \multicolumn{5}{|c|}{ L 1} & \multicolumn{5}{|c|}{ L 2} \\
\hline & & H 1 & H 2 & H 3 & H 4 & H 5 & H 1 & H 2 & H 3 & H 4 & H 5 \\
\hline \multirow{9}{*}{ O 1} & \multirow{3}{*}{ C 1} & 3.00 & 1.85 & 0.75 & 1.35 & 1.45 & 1.80 & 0.70 & 2.50 & 1.70 & 0.40 \\
\hline & & 2.85 & 1.75 & 0.90 & 1.40 & 1.40 & 1.85 & 0.75 & 2.55 & 1.75 & 0.50 \\
\hline & & 3.05 & 1.90 & 0.85 & 1.30 & 1.50 & 1.90 & 0.80 & 2.65 & 1.80 & 0.45 \\
\hline & \multirow{3}{*}{ C 2} & 1.00 & 1.10 & 1.00 & 1.60 & 1.50 & 1.60 & 1.75 & 0.50 & 1.35 & 1.10 \\
\hline & & 1.15 & 1.20 & 1.05 & 1.65 & 1.55 & 1.50 & 1.70 & 0.55 & 1.30 & 1.15 \\
\hline & & 0.95 & 0.95 & 1.10 & 1.50 & 1.60 & 1.55 & 1.75 & 0.60 & 1.40 & 1.20 \\
\hline & \multirow{3}{*}{ C 3} & 1.10 & 1.50 & 1.80 & 1.45 & 1.25 & 0.85 & 0.65 & 0.55 & 0.90 & 0.90 \\
\hline & & 1.05 & 1.55 & 1.85 & 1.50 & 1.20 & 0.90 & 0.60 & 0.45 & 0.95 & 0.95 \\
\hline & & 1.10 & 1.60 & 1.70 & 1.55 & 1.35 & 0.95 & 0.55 & 0.60 & 1.00 & 1.00 \\
\hline \multirow{9}{*}{ O 2} & \multirow{3}{*}{ C 1} & 1.75 & 3.50 & 2.50 & 2.00 & 0.65 & 2.00 & 3.00 & 2.55 & 3.00 & 2.65 \\
\hline & & 1.80 & 3.45 & 2.55 & 2.10 & 0.70 & 2.05 & 3.05 & 2.50 & 3.05 & 2.60 \\
\hline & & 1.70 & 3.40 & 2.60 & 2.15 & 0.55 & 2.00 & 2.95 & 2.45 & 2.90 & 2.55 \\
\hline & \multirow{3}{*}{ C 2} & 1.10 & 1.05 & 0.50 & 1.05 & 1.25 & 1.20 & 1.35 & 1.20 & 0.30 & 2.50 \\
\hline & & 1.15 & 1.00 & 0.60 & 1.00 & 1.30 & 1.25 & 1.30 & 1.30 & 0.40 & 2.55 \\
\hline & & 1.00 & 1.05 & 0.65 & 1.10 & 1.35 & 1.35 & 1.25 & 1.40 & 0.45 & 2.60 \\
\hline & \multirow{3}{*}{ C 3} & 1.05 & 1.25 & 2.00 & 1.50 & 2.10 & 1.00 & 2.70 & 2.15 & 2.10 & 2.70 \\
\hline & & 1.00 & 1.20 & 2.05 & 1.60 & 2.05 & 0.90 & 2.60 & 2.00 & 2.00 & 2.75 \\
\hline & & 1.10 & 1.35 & 2.20 & 1.65 & 2.00 & 1.05 & 2.55 & 2.10 & 2.05 & 2.80 \\
\hline
\end{tabular}

first group, and $a_{2}(1)=2$ and $a_{2}(2)=5$ "active" levels for the second group. We have $n_{1}=2 \times 3=6$ treatments in the first group and $n_{2}=2+5=7$ treatments in the second group. So we have $n=n_{1} \times n_{2} \times r=6 \times 7 \times 3=126$ observations. The selected observations are presented in Tables 5.3 and 5.4

The parameters of the model will be associated to the $\mathbf{h}=\left(h_{1}, h_{2}\right)$ vectors of $\Gamma=\left\{\mathbf{h}: 0 \leq h_{l} \leq u_{l}, \quad l=1,2\right\}$.

For the first group we have matrices $\mathbf{A}_{1}(k)$, with $k=1,2$, defined by

$$
\left\{\begin{array}{l}
\mathbf{A}_{1}(0)=\frac{1}{\sqrt{2}}\left(\mathbf{1}^{2}\right)^{\prime} \otimes \frac{1}{\sqrt{3}}\left(\mathbf{1}^{3}\right)^{\prime} \\
\mathbf{A}_{1}(1)=\mathbf{T}_{2} \otimes \frac{1}{\sqrt{3}}\left(\mathbf{1}^{3}\right)^{\prime} \\
\mathbf{A}_{1}(2)=\mathbf{I}_{2} \otimes \mathbf{T}_{3},
\end{array}\right.
$$

with

$$
\mathbf{T}_{2}=\left[\begin{array}{ll}
\frac{\sqrt{2}}{2} & -\frac{\sqrt{2}}{2}
\end{array}\right]
$$


TABLE 5.2

Production in $\mathrm{kg}$ per plant - observations.

\begin{tabular}{|c|c|c|c|c|c|c|}
\hline & \multicolumn{5}{|c|}{ L 3} \\
\hline & & H 1 & H 2 & H 3 & $\mathrm{H} 4$ & H 5 \\
\hline \multirow{9}{*}{ O 1} & \multirow{3}{*}{ C 1} & 1.05 & 1.50 & 1.15 & 0.85 & 1.15 \\
\hline & & 1.10 & 1.60 & 1.20 & 0.70 & 1.10 \\
\hline & & 1.15 & 1.45 & 1.10 & 0.95 & 1.05 \\
\hline & \multirow{3}{*}{ C 2} & 0.75 & 0.65 & 0.90 & 0.85 & 1.05 \\
\hline & & 0.80 & 0.60 & 0.95 & 0.95 & 1.10 \\
\hline & & 0.95 & 0.75 & 1.10 & 1.00 & 1.15 \\
\hline & \multirow{3}{*}{ C 3} & 0.90 & 0.90 & 0.55 & 0.70 & 0.35 \\
\hline & & 0.95 & 0.95 & 0.75 & 0.80 & 0.45 \\
\hline & & 0.90 & 1.05 & 0.60 & 0.65 & 0.40 \\
\hline \multirow{9}{*}{ O 2} & \multirow{3}{*}{ C 1} & 1.60 & 3.05 & 0.25 & 1.65 & 2.65 \\
\hline & & 1.65 & 3.00 & 0.35 & 1.70 & 2.50 \\
\hline & & 1.60 & 2.95 & 0.40 & 1.75 & 2.70 \\
\hline & \multirow{3}{*}{ C 2} & 1.05 & 1.95 & 2.00 & 2.20 & 2.35 \\
\hline & & 1.10 & 1.90 & 2.05 & 2.30 & 2.40 \\
\hline & & 1.15 & 2.00 & 2.15 & 2.15 & 2.45 \\
\hline & \multirow{3}{*}{ C 3} & 1.60 & 1.10 & 2.05 & 1.50 & 3.00 \\
\hline & & 1.65 & 1.15 & 2.20 & 1.60 & 2.90 \\
\hline & & 1.75 & 1.25 & 1.95 & 1.65 & 3.15 \\
\hline
\end{tabular}

and

$$
\mathbf{T}_{3}=\left[\begin{array}{ccc}
\frac{2 \sqrt{6}}{6} & -\frac{\sqrt{6}}{6} & -\frac{\sqrt{6}}{6} \\
0 & \frac{\sqrt{2}}{2} & -\frac{\sqrt{2}}{2}
\end{array}\right]
$$

For the second group we have matrices $\mathbf{A}_{2, j}(k)$, with $k=1,2$ and $j=1,2$, defined by

$$
\left\{\begin{array}{l}
\mathbf{A}_{2,1}(1)=D\left[\frac{1}{\sqrt{2}}\left(\mathbf{1}^{2}\right)^{\prime},\left(\mathbf{0}^{5}\right)^{\prime}\right] \\
\mathbf{A}_{2,2}(1)=D\left[\mathbf{T}_{2},\left(\mathbf{0}^{5}\right)^{\prime}\right] \\
\mathbf{A}_{2,1}(2)=D\left[\left(\mathbf{0}^{2}\right)^{\prime}, \frac{1}{\sqrt{5}}\left(\mathbf{1}^{5}\right)^{\prime}\right] \\
\mathbf{A}_{2,2}(2)=D\left[\left(\mathbf{0}^{2}\right)^{\prime}, \mathbf{T}_{5}\right],
\end{array}\right.
$$


TABLE 5.3

Production in $\mathrm{kg}$ per plant - selected observations.

\begin{tabular}{|c|c|c|c|c|c|c|c|c|c|c|c|}
\hline & \multicolumn{5}{|c|}{ L 1} & \multicolumn{5}{|c|}{ L 2} \\
\hline & & H 1 & H 2 & H 3 & H 4 & H 5 & H 1 & H 2 & H 3 & H 4 & H 5 \\
\hline \multirow{9}{*}{ O 1} & \multirow{3}{*}{ C 1} & 3.00 & 1.85 & 0.75 & 1.35 & 1.45 & & & & 1.70 & \\
\hline & & 2.85 & 1.75 & 0.90 & 1.40 & 1.40 & & & & 1.75 & \\
\hline & & 3.05 & 1.90 & 0.85 & 1.30 & 1.50 & & & & 1.80 & \\
\hline & \multirow{3}{*}{ C 2} & 1.00 & 1.10 & 1.00 & 1.60 & 1.50 & & & & 1.35 & \\
\hline & & 1.15 & 1.20 & 1.05 & 1.65 & 1.55 & & & & 1.30 & \\
\hline & & 0.95 & 0.95 & 1.10 & 1.50 & 1.60 & & & & 1.40 & \\
\hline & \multirow{3}{*}{ C 3} & 1.10 & 1.50 & 1.80 & 1.45 & 1.25 & & & & 0.90 & \\
\hline & & 1.05 & 1.55 & 1.85 & 1.50 & 1.20 & & & & 0.95 & \\
\hline & & 1.10 & 1.60 & 1.70 & 1.55 & 1.35 & & & & 1.00 & \\
\hline \multirow{9}{*}{ O 2} & \multirow{3}{*}{ C 1} & 1.75 & 3.50 & 2.50 & 2.00 & 0.65 & & & & 3.00 & \\
\hline & & 1.80 & 3.45 & 2.55 & 2.10 & 0.70 & & & & 3.05 & \\
\hline & & 1.70 & 3.40 & 2.60 & 2.15 & 0.55 & & & & 2.90 & \\
\hline & \multirow{3}{*}{ C 2} & 1.10 & 1.05 & 0.50 & 1.05 & 1.25 & & & & 0.30 & \\
\hline & & 1.15 & 1.00 & 0.60 & 1.00 & 1.30 & & & & 0.40 & \\
\hline & & 1.00 & 1.05 & 0.65 & 1.10 & 1.35 & & & & 0.45 & \\
\hline & \multirow{3}{*}{ C 3} & 1.05 & 1.25 & 2.00 & 1.50 & 2.10 & & & & 2.10 & \\
\hline & & 1.00 & 1.20 & 2.05 & 1.60 & 2.05 & & & & 2.00 & \\
\hline & & 1.10 & 1.35 & 2.20 & 1.65 & 2.00 & & & & 2.05 & \\
\hline
\end{tabular}

with

$$
\mathbf{T}_{5}=\left[\begin{array}{rrrrr}
\frac{2 \sqrt{5}}{5} & -\frac{\sqrt{5}}{10} & -\frac{\sqrt{5}}{10} & -\frac{\sqrt{5}}{10} & -\frac{\sqrt{5}}{10} \\
0 & \frac{\sqrt{3}}{2} & -\frac{\sqrt{3}}{6} & -\frac{\sqrt{3}}{6} & -\frac{\sqrt{3}}{6} \\
0 & 0 & \frac{\sqrt{6}}{3} & -\frac{\sqrt{6}}{6} & -\frac{\sqrt{6}}{6} \\
0 & 0 & 0 & \frac{\sqrt{2}}{2} & -\frac{\sqrt{2}}{2}
\end{array}\right]
$$

When we cross the both groups we obtain the model in its canonical form as

$$
\mathbf{y}=\sum_{\mathbf{h} \in \Gamma} \mathbf{A}(\mathbf{h})^{\prime} \eta(\mathbf{h})+\mathbf{A}^{\perp} \mathbf{e}
$$


TABLE 5.4

Production in $\mathrm{kg}$ per plant - selected observations.

\begin{tabular}{|c|c|c|c|c|c|c|}
\hline & \multicolumn{5}{|c|}{ L 3} \\
\hline & & H 1 & $\mathrm{H}_{2}$ & H 3 & $\mathrm{H} 4$ & H 5 \\
\hline \multirow{9}{*}{ O 1} & \multirow{3}{*}{ C 1} & & & & & 1.15 \\
\hline & & & & & & 1.10 \\
\hline & & & & & & 1.05 \\
\hline & \multirow{3}{*}{ C 2} & & & & & 1.05 \\
\hline & & & & & & 1.10 \\
\hline & & & & & & 1.15 \\
\hline & \multirow{3}{*}{ C 3} & & & & & 0.35 \\
\hline & & & & & & 0.45 \\
\hline & & & & & & 0.40 \\
\hline \multirow{9}{*}{ O 2} & \multirow{3}{*}{ C 1} & & & & & 2.65 \\
\hline & & & & & & 2.50 \\
\hline & & & & & & 2.70 \\
\hline & \multirow{3}{*}{ C 2} & & & & & 2.35 \\
\hline & & & & & & 2.40 \\
\hline & & & & & & 2.45 \\
\hline & \multirow{3}{*}{ C 3} & & & & & 3.00 \\
\hline & & & & & & 2.90 \\
\hline & & & & & & 3.15 \\
\hline
\end{tabular}

where matrices $\mathbf{A}(\mathbf{h})$ are defined as

$$
\left\{\begin{array}{l}
\mathbf{A}(0,0)=\mathbf{A}_{1}(0,1)+\mathbf{A}_{1}(0,2) \\
\mathbf{A}(i, 0)=\mathbf{A}_{1}(i, 1)+\mathbf{A}_{1}(i, 2), \quad i=1,2 \\
\mathbf{A}(0, l)=\mathbf{A}_{2}(0, l), \quad l=1,2 \\
\mathbf{A}(i, 1)=\mathbf{A}_{2}(i, 1), \quad i=1,2 \\
\mathbf{A}(i, 2)=\mathbf{A}_{2}(i, 2), \quad i=1,2,
\end{array}\right.
$$

with matrices

$$
\mathbf{A}_{j}\left(k_{1}, k_{2}\right)=\mathbf{A}_{1}\left(k_{1}\right) \otimes \mathbf{A}_{2, j}\left(k_{2}\right) \otimes \frac{1}{\sqrt{3}}\left(\mathbf{1}^{3}\right)^{\prime},
$$

for $j=1,2, k_{1}=0,1,2$ and $k_{2}=1,2$. Matrix $\mathbf{A}^{\perp}$ is given by

$$
\mathbf{A}^{\perp}=\mathbf{I}_{42} \otimes \mathbf{T}_{3} .
$$


TABLE 5.5

Results for the cross of balanced nesting and stair nesting.

\begin{tabular}{cccc}
\hline $\mathbf{h}$ & \multicolumn{1}{c}{$S(\mathbf{h})$} & $g(\mathbf{h})$ & $\widetilde{\gamma}(\mathbf{h})$ \\
\hline$(1,0)$ & 0.8054 & 2 & 0.4027 \\
$(2,0)$ & 5.6226 & 8 & 0.7028 \\
$(0,1)$ & 0.9344 & 1 & 0.9344 \\
$(0,2)$ & 1.3971 & 4 & 0.3493 \\
$(1,1)$ & 0.0711 & 1 & 0.0711 \\
$(1,2)$ & 7.3929 & 4 & 1.8482 \\
$(2,1)$ & 9.6086 & 4 & 2.4022 \\
$(2,2)$ & 38.8077 & 16 & 2.4255 \\
\hline
\end{tabular}

TABLE 5.6

Results for the cross of balanced nesting and stair nesting.

\begin{tabular}{ccc}
\hline$S$ & $g$ & $\widetilde{\sigma}^{2}$ \\
\hline 0.4100 & 84 & 0.0049 \\
\hline
\end{tabular}

In Tables 5.5 and 5.6 we present the results for the cross of balanced nesting and stair nesting.

The variances of these estimators are given by

$$
\operatorname{Var}[\widetilde{\gamma}(\mathbf{h})]=\operatorname{Var}\left[\frac{S(\mathbf{h})}{g(\mathbf{h})}\right]=\frac{2 \gamma^{2}(\mathbf{h})}{g(\mathbf{h})} .
$$


So $\widetilde{\operatorname{Var}}[\widetilde{\gamma}(\mathbf{h})]=\frac{2 \widetilde{\gamma}^{2}(\mathbf{h})}{g(\mathbf{h})}$. For this case we have

$$
\left\{\begin{array}{l}
\widetilde{\operatorname{Var}}[\widetilde{\gamma}(1,0)]=0.1621850 \\
\widetilde{\operatorname{Var}}[\widetilde{\gamma}(2,0)]=0.1234910 \\
\widetilde{\operatorname{Var}}[\widetilde{\gamma}(0,1)]=1.7463700 \\
\widetilde{\operatorname{Var}}[\widetilde{\gamma}(0,2)]=0.0609975 \\
\widetilde{\operatorname{Var}}[\widetilde{\gamma}(1,1)]=0.0101136 \\
\widetilde{\operatorname{Var}}[\widetilde{\gamma}(1,2)]=1.7079600 \\
\widetilde{\operatorname{Var}}[\widetilde{\gamma}(2,1)]=2.8851700 \\
\widetilde{\operatorname{Var}}[\widetilde{\gamma}(2,2)]=0.7353690 \\
\widetilde{\operatorname{Var}}\left[\widetilde{\sigma}^{2}\right]=0.0000005 .
\end{array}\right.
$$

6. Final Comments. Stair nested designs may be very useful since they require fewer observations than the usual balanced nested designs and with the stair nested designs we can have a good distribution of the degrees of freedom among the variance components. Staggered nested designs have the same advantages but they are not orthogonal, unlike the stair nested designs. Furthermore it is more easy to carry out inference for stair nesting than for staggered nesting since the stair nesting holds the simplicity of the algebraic structure of balanced nesting, unlike the staggered nesting.

the cross between balanced and stair nested designs require fewer observations than the usual cross between balanced nested designs since when we cross balanced and stair nesting, only 126 observations are required, less that the 270 ones required for cross of balanced nesting. This is in fact a big advantage when we compare both studies since the cross of balanced nesting and stair nesting will allow experiments that will become cheaper, due to the fewer number of observations involved, or with the same resources we produce more experiments. Since in a practical experiment, the implementation cost is, many times, a decisive factor, the cross balanced and stair nesting will be a strong alternative to the cross of balanced nesting. Moreover, for the cross of balanced and stair nesting it is easy to carry out inference because it is very similar to the cross of balanced nesting, that is well studied. 
Acknowledgements. This work was partially supported by the Fundação para a Ciência e a Tecnologia (Portuguese Foundation for Science and Technology) through PEst-OE/MAT/UI0297/2011 (CMA).

We thank the referees and editors who have reviewed this article for their constructive suggestions.

\section{REFERENCES}

[1] R. Bailey. Association Schemes. Designed Experiments, Algebra and Combinatorics. Cambridge University Press, Cambridge, 2004.

[2] R. Bailey. Design of Comparactive Experiments. Cambridge University Press, Cambridge, 2008.

[3] T. Bainbridge. Staggered nested designs for estimating variance components. Industrial Quality Control, 22:12-20, 1965.

[4] D. Cox and P. Solomon. Components of Variance. Chapman and Hall, New York, 2003.

[5] C. Fernandes Models with cross step nesting. PhD Dissertation, Universidade da Beira Interior, 2009.

[6] C. Fernandes, P. Ramos and J. Mexia. Algebraic structure of step nesting designs. Discussiones Mathematicae - Probability and Statistics, 30:221-235, 2010.

[7] M. Fonseca, J. Mexia and R. Zmyślony. Estimators and tests for variance components in cross nested orthogonal designs. Discussiones Mathematicae - Probability and Statistics, 23:175$201,2003$.

[8] M. Fonseca, J. Mexia and R. Zmyślony. Binary Operations on Jordan Algebras and Orthogonal Normal Models. Linear Algebra and its Applications, 417:75-86, 2006.

[9] S. Gilmour and P. Goos. Analysis of data from non-orthogonal multistratum designs in industrial experiments. Journal of the Royal Statistical Society, Series C (Applied Statistics), 58:467484, 2009.

[10] D. Janky. Sometimes pooling for analysis of variance hypothesis tests: A review and study of a split-plot model. The American Statistician, 54:269-279, 2000.

[11] R. Khattree and D. Naik. Statistical tests for random effects in staggered nested designs. Journal of Applied Statistics, 22:495-505, 1995.

[12] R. Khattree, D. Naik and R. Mason. Estimation of variance components in staggered nested designs. Journal of Applied Statistics, 24:395-408, 1997.

[13] A. Khuri, T. Mathew and B. Sinha. Statistical tests for mixed linear models. John Wiley and Sons, New York, 1998.

[14] E. Lehmann. Testing statistical hypotheses. John Wiley and Sons, New York, 1959.

[15] F. Leone, L. Nelson, N. Johnson and S. Eisenstat. Sampling distributions of variance components II. Empirical studies of unbalanced nested designs. Technometrics, 10:719-737, 1968.

[16] R. Mason, R. Gunst and J. Hess. Statistical Design and Analysis of Experiments. John Wiley and Sons, New York, 1989.

[17] D. Montgomery. Design and Analysis of Experiments, 6th ed. John Wiley and Sons, New York, 2004.

[18] D. Naik and R. Khattree. A computer program to estimate variance components in staggered nested designs. Journal of Quality Technology, 30:292-297, 1998.

[19] J. Nelder. The interpretation of negative components of variance. Biometrika, 41:544-548, 1954.

[20] L. Nelson. Variance estimation using staggered nested designs. Journal of Quality Technology, 15:195-198, 1983.

[21] L. Nelson. Using nested designs. 1. Estimation of standard deviations. Journal of Quality Technology, 27:169-171, 1995. 
[22] L. Nelson. Using nested designs. 2. Confidence limits for standard deviations. Journal of Quality Technology, 27:265-267, 1995.

[23] Y. Ojima. General formulae for expectations, variances and covariances of the mean squares for staggered nested designs. Journal of Applied Statistics, 25:785-799, 1998.

[24] Y. Ojima. Generalized staggered nested designs for variance components estimation. Journal of Applied Statistics, 27:541-553, 2000.

[25] H. Sahai and M. Ojeda. Analysis of Variance for Random Models. Volume I: Balanced Data. Birkhäuser, Boston, 2004.

[26] S. Searle, G. Casella and C. McCulloch. Variance Components. John Wiley and Sons, New York, 1992.

[27] G. Senedcor and W. Cochran. Statistical Method, 8th ed. Iowa State University Press, Ames, Iowa, 1989.

[28] S. Silvey. Statistical Inference. CRC Monographs on Statistics and Applied Probability, Chapman and Hall, 1975.

[29] J. Smith and J. Beverly. The use and analysis of staggered nested factorial designs. Journal of Quality Technology, 13:166-173, 1981. 